Original Research Article

\title{
Quality of prescriptions in hospitalized children suffering from acute and persistent diarrhoea
}

\author{
Nazuk Sharma ${ }^{1 *}$, D. C. Dhasmana ${ }^{1}$, Taruna Sharma ${ }^{1}$, Alpa Gupta ${ }^{2}$
}

\begin{abstract}
${ }^{1}$ Department of Pharmacology, ${ }^{2}$ Department of Paediatrics, Himalayan Institute of Medical Sciences, Jolly grants, Dehradun, Uttarakhand, India
\end{abstract}

Received: 07 February 2019

Accepted: 14 March 2019

*Correspondence to:

Dr. Nazuk Sharma,

Email: nazuk89@yahoo.com

Copyright: (C) the author(s), publisher and licensee Medip Academy. This is an openaccess article distributed under the terms of the Creative Commons Attribution NonCommercial License, which permits unrestricted noncommercial use, distribution, and reproduction in any medium, provided the original work is properly cited.

\begin{abstract}
Background: Diarrhoea is a major public health problem in children worldwide. It continues to be a major health challenge, especially in developing countries, despite the availability of regularly updated standard treatment guidelines. Noncompliance to such guidelines by the physicians has been a long standing story. The treatment is often marred with incapacitating prescription of drugs besides neglecting even the basic tenets of good prescribing. As a result, the quality of such prescriptions for diarrhoeal disorders in children remains poor. To gauge the magnitude of this problem in this setup towards possible corrective measures, the study was aimed to audit prescription practices in the management of acute and persistent diarrhoea in hospitalised children up to five years of age.

Methods: An observational study was conducted in 100 patients of either gender in the age group up to 5 years admitted with acute and persistent diarrhoea. A detailed medical history from the parents/guardians and the details of prescription from the time of admission till the discharge of the patient were obtained. Quality of prescriptions was analysed using prescription quality index (PQI) tool, a validated comprehensive tool described by Hassan et al in 2010. Based on this tool, prescription with the total PQI score of $\leq 31$ were interpreted as poor quality, scores with 32 to 33 as medium quality and scores 34 to 43 as high quality with a possible maximum score of ' 43 '.

Results: Based on the PQI tool for 100 children, 60 prescriptions were found to be of poor quality. Only 2 prescriptions were of medium quality, whereas 38 prescriptions were in high quality range. Average mean \pm SD score of prescriptions with poor quality was $25.2 \pm 1.48$, ranging from 21 to 31 . The mean \pm SD of prescriptions with medium quality was observed to be $32 \pm 0$ and for prescriptions of high quality was $38.07 \pm 2.28$. The total average mean score of all prescriptions was $30.23 \pm 6.50$. Poor quality prescriptions were particularly observed for the patients with the diarrhoea with No dehydration.

Conclusions: Prescription appropriateness in spite of available guidelines continues to be a big challenge in the adequate management of patients with diarrhoeal disorders under the age group of five years in a tertiary care centre in India.
\end{abstract}

Keywords: Children, Diarrhoea, Prescription Quality Index tool, Quality of prescriptions

\section{INTRODUCTION}

Diarrhoea remains a common illness among infants and children throughout the world making it a leading cause of childhood morbidity and mortality. In developing countries it is the second highest cause of death in children up to five years of age after pneumonia. ${ }^{1}$ In 2016, it accounted for $9 \%$ of deaths due to diarrhoea in this age group in India. ${ }^{2}$ The situation in the state of Uttarakhand is 
abysmal with the $17 \%$ prevalence of diarrhoeal disorders as compared to overall Indian prevalence of $9.2 \% .^{3}$

World health organisation (WHO) defines acute diarrhoea as the passage of unusually loose or watery stools, usually at least three times in a 24 hour period and persistent diarrhoea that lasts for 14 days or longer. Although, the mild illness usually resolves without treatment, but varying degree of water and electrolytes loss occurs during the episodes which need prompt action to protect the health of the child. ${ }^{4}$

For treatment of diarrhoeal disorders, regularly updated standard guidelines are provided by WHO which clearly elaborates the management protocol for each type of the presentation along with all do's and don'ts. A significant progress was achieved with the introduction of reducedosmolarity oral rehydration solution (ORS) and zinc supplementation as a part of the management of diarrhoeal disorders in children. ${ }^{4}$ However, these guidelines are often flawed and ignored in routine practice. Doctor's are usually apprehensive, more concerned for young baby that at times makes them unwilling to accept the standard guidelines. Moreover, the disappointing fact is that doctors fail to keep up the pace with changing guidelines on diarrhoea with the impression that the guidelines are too simple. Further parental desire of 'real' drug therapy to pressurise the physician to stop diarrhoea are some of the blatant reasons for poor quality of prescriptions in this setting. ${ }^{5}$ Even in India, even the routine use of ORS and zinc supplementation is still not practiced widely. ${ }^{3}$

In fact, inappropriate drug prescribing is a global problem despite the availability of standard guidelines. Currently, accurate prescribing decisions, rational use of drugs appropriate treatment and associated patient-instructions, are of major concerns in management of a patient. Realising the magnitude of the problem, the aim of the study was to conduct an audit of prescription practices in the management of acute and persistent diarrhoea in hospitalised children up to five years of age.

\section{METHODS}

An observational descriptive study was conducted from January 2017 to December 2017 to analyse the prescribing patterns in the management of acute and persistent diarrhoea in hospitalized children. Patients were included in the study after obtaining written informed consent from parents/guardians and approval from the institutional ethics committee. A total of 100 patients of either gender in the age group up to 5 years admitted with acute and persistent diarrhoea were included in the study. Patients with concurrent severe illness, underlying septicaemia, metabolic disorders or any other major organ failure were excluded. A detailed medical history was obtained from parents/guardians. All the relevant information including history, physical examination, investigations and treatment along with discharge summary were recorded from the time of admission till the discharge of the patient, taken together as a single prescription for convenience of analysis. Follow up was done up to $28^{\text {th }}$ day following admission. Compliance to treatment given at home was ascertained at repeated intervals. Prescriptions were analysed for adherence to the WHO standard guidelines put forward in 2005 for the management of diarrhoea in children up to 5 years of age.

The evaluation of quality of prescription was done using prescription quality index (PQI) tool, recently described by Hassan et al in 2010.The earlier WHO core prescribing indicators that measures only some dimensions that were mutually agreed upon to take care of majority of the countries, of appropriateness of drug prescribing behaviour, but not necessarily the important ones and to look for potential problems and possible corrections. ${ }^{6,7}$ This include only the average number of drugs prescribed, utilisation of drugs from essential drug list, prescribing with generic names, the percentage encounters with the antimicrobials and injectable in a prescription. Due to this inherent problem WHO core prescribing indicators are not a gold standard for rational prescribing. The PQI tool is an attempt to overcome the drawbacks of WHO core indicators, that was introduced as a more comprehensive tool encompassing drug related verification of correct indication, dosage, administration, their effectiveness with supportive evidence, duration of therapy, cost analysis, legibility of prescription and prescribers information along with adequate patient information, provision for possible drug-drug or drug-disease interaction, duplication of drugs, adverse drug reaction, and finally assessing whether the prescription fulfilled the patients requirement of drug therapy.

PQI tool mentions 22 criteria's for the above parameters in the form of questions. The scores in the PQI tool for each criteria varied as ' 0 ' to ' 4 ' for very important criteria ( $\mathrm{n}=$ $2)$, ' 0 ' to ' 2 ' for criteria considered as important $(n=15)$, and ' 0 ' to ' 1 ' for less important criteria $(n=5)$,. Prescriptions usually included more than one drug, hence each drug was rated individually. If a drug was not indicated, criteria 1 was scored as ' 0 ', subsequently criteria 2 (dosage), criteria 11 (duration) and criteria 12 (cost) were all scored as ' 0 '. The individual PQI score was drawn for each drug in the prescription. The total PQI score for the prescription was calculated by adding minimum scores achieved for each criterion by the drugs. The possible maximum score of PQI was '43'. Prescription with the total PQI score of $\leq 31$ was interpreted as poor quality, scores with 32 to 33 as medium quality and scores 34 to 43 as high quality as described by the PQI. The manual for the PQI includes an introduction to the PQI, a listing of the 22 criteria, steps on how to use the PQI, specific instructions with an operational definition of the terms, a scoring method for each criterion, and the assessment form to be used for the prescription rating. ${ }^{6}$

The data was collected and entered in MS Excel 2010. The one-sample Kolmogorov-Smirnov Test was employed to determine whether the data sets differed from a normal 
distribution. Descriptive statistics was calculated for quantitative data. Frequency and percentage were calculated for qualitative data and presented in the form of graphs and tables.

\section{RESULTS}

This study was done to analyse the prescribing patterns in the management of acute and persistent diarrhoea in hospitalized children up to 5 years of age. Out of the total 100 hospitalised patients 32 were females and 68 were males and followed for the period of their stay in the hospital. Median age of children was observed to be 14 months. Most of the children admitted were below the age of one year while almost $81 \%$ patients were below two years of age. Admissions were more from people who were from urban areas than rural areas. The parents were relatively more from poverty stricken status (Table 1).

Table 1: Demographic profile of the patients admitted with acute and persistent diarrhoea up to 5 years of age $(n=100)$.

\begin{tabular}{|lll|}
\hline $\begin{array}{l}\text { Demographic } \\
\text { profile }\end{array}$ & $\begin{array}{l}\text { Subgroup } \\
\text { Age-group }\end{array}$ & $\begin{array}{l}\text { Frequency } \\
(\%)\end{array}$ \\
\cline { 2 - 3 } & 1-28 days & $4(4.0 \%)$ \\
\cline { 2 - 3 } & 7-12 months & $22(22.0 \%)$ \\
\cline { 2 - 3 } & 13-24 months & $21(21.0 \%)$ \\
\cline { 2 - 3 }$>25$ months & $34(34.0 \%)$ \\
\hline \multirow{2}{*}{ Sex } & Female & $32(32.0 \%)$ \\
\hline \multirow{2}{*}{ Residence } & Male & $68(68.0 \%)$ \\
\hline \multirow{2}{*}{ Parent occupation } & Rural & $47(47.0 \%)$ \\
\cline { 2 - 3 } & Urban & $53(53.0 \%)$ \\
\cline { 2 - 3 } & Self employed & $38(38.0 \%)$ \\
\cline { 2 - 3 } & Local vendor & $57(57.0 \%)$ \\
\hline
\end{tabular}

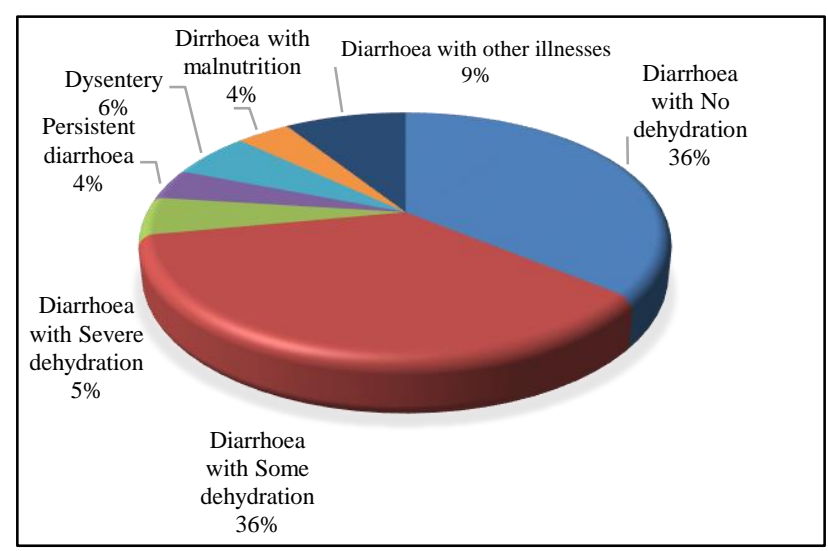

Data expressed in percentage

Figure 1: Distribution of diagnosis of the patients admitted with diarrhoea up to 5 years of age $(n=100)$.

Children presented with varying degree of dehydration status (Figure 1). Equal numbers of patients were observed with no and some degree of dehydration, with only $5 \%$ cases of severe degree of dehydration. Below $10 \%$ admissions were of the cases of persistent diarrhoea and dysentery. Underlying malnutrition with diarrhoea was observed in $4 \%$ cases. The average duration for which the child was admitted was $6.630 \pm 4.101$ days with minimum one day of admission to up to a maximum of 21 days.

WHO guidelines recommend the use of ORS and zinc for all children. It was observed that ORS and zinc was used only in $62 \%$ and $81 \%$ of children respectively. Subgroup analysis of use of ORS and zinc was done. It was observed that all patients of persistent diarrhoea received ORS and zinc. Use of zinc was more frequent than the ORS. Surprisingly use of ORS and zinc was comparatively less in patients with no and some dehydration.

Antimicrobials were commonly prescribed in the study patients. From a total of 100 patients 97 patients received one or more antibiotics, out of which at least one antibiotic was prescribed to 61 patients, two antibiotics to 23 patients and three antibiotics to 12 patients. One patient was prescribed upto four antibiotics. Among the antibiotics prescribed, Cephalosporin's were the most commonly prescribed class followed by Aminoglycosides and Penicillins. Various other drugs were also prescribed during the hospital stay of children (Table 2). Antiemetic use was seen invariably in $50 \%$ of the prescriptions. Use of prebiotics and probiotics was frequently seen $(43 \%)$. Multivitamin combinations were prescribed in almost $20 \%$ of the cases. Miscellaneous drugs included the drugs used for the management of associated complaints like perianal ulceration, anaemia, urinary tract infections etc.

Training of the mother has been the key focus in management of diarrhoea in children and well emphasized with documentation. Instructions for the use of ORS were documented in $62 \%$ prescriptions. Written documentation for encouragement of oral fluids and breast feeding during the episodes was observed only in $3 \%$ and $11 \%$ patients respectively.

Prescriptions were further evaluated according to the PQI tool as depicted in table 3. Out of a total of 100 prescriptions 60 prescriptions were found to be of poor quality. Only 2 prescriptions coming under medium quality, whereas 38 prescriptions were found to be of high quality. Average mean $\pm \mathrm{SD}$ of prescriptions with poor quality was $25.2 \pm 1.48$, (range $21-31$ ), for medium quality 32 (range 32-33) and for high quality was $38.07 \pm 2.28$ (range 34-43). The total average mean score of all prescriptions was $30.23 \pm 6.50$. Maximum poor quality prescriptions were observed for the patients with the diarrhoea with No dehydration (Table 3). Out of the total of 22 criteria's of PQI tool each criteria was separately analysed as expressed in Table 4 with the maximum score allotted to each question. It was observed that the mean score obtained for criteria one, three, four, ten, eleven, twelve, thirteen, fourteen and twenty-one were less than $50 \%$ of their maximum which reflects the reasons for the poor quality of the prescription. 
Table 2: Distribution of other drugs used and pattern of prescribing in patients with acute and persistent diarrhoea up to 5 years of age $(n=100)$.

\begin{tabular}{|llll|}
\hline Drugs & $\begin{array}{l}\text { Prescribed } \\
\text { Prescribed with } \\
\text { generic name }\end{array}$ & $\begin{array}{l}\text { Prescribed with } \\
\text { brand name }\end{array}$ & Rationality behind prescribing \\
\hline Antimicrobial drugs & 0 & 97 & $\begin{array}{l}\text { 40 patients had no absolute indication } \\
\text { At least one antimicrobial was prescribed in Brand } \\
\text { name in each prescription }\end{array}$ \\
\hline Antipyretic drugs & 1 & 46 & 23 patients were recorded to have temperature \\
\hline Antiemetic drugs & 0 & 50 & Not recommended as per the WHO guidelines \\
\hline Antacids drugs & 0 & 58 & Not recommended as per the WHO guidelines \\
\hline Pre/probiotics & 0 & 43 & Not recommended as per the WHO guidelines \\
\hline Multivitamins & 0 & 20 & $\begin{array}{l}\text { 2 patients of malnutrition were prescribed } \\
\text { multivitamins rationally. }\end{array}$ \\
\hline Anti-diarrhoeal & 1 & 5 & Not recommended as per the WHO guidelines \\
\hline Miscellaneous & 0 & 58 & $\begin{array}{l}\text { For use of perianal ulceration, anaemia, urinary tract } \\
\text { infections }\end{array}$ \\
\hline
\end{tabular}

Table 3: Quality of prescriptions in patients with acute and persistent diarrhoea up to 5 years of age according to PQI scores $(\mathbf{n}=\mathbf{1 0 0})$.

\begin{tabular}{|llllllll|}
\hline & $\begin{array}{l}\text { Poor } \\
\text { quality } \\
\mathbf{3 1}\end{array}$ & $\mathbf{\%}$ & $\begin{array}{l}\text { Medium } \\
\text { quality } \\
\mathbf{3 2 - 3 3}\end{array}$ & $\mathbf{\%}$ & $\begin{array}{l}\text { High } \\
\text { quality } \\
\mathbf{3 4 - 4 3}\end{array}$ & $\begin{array}{l}\text { \% } \\
\text { Total }\end{array}$ \\
\hline Diarrhoea with no dehydration & 30 & $83.30 \%$ & 0 & $0.00 \%$ & 6 & $16.70 \%$ & 36 \\
\hline Diarrhoea with some dehydration & 20 & $55.60 \%$ & 2 & $5.60 \%$ & 14 & $38.90 \%$ & 36 \\
\hline Diarrhoea with Severe dehydration & 1 & $20.00 \%$ & 0 & $0.00 \%$ & 4 & $80.00 \%$ & 5 \\
\hline Persistent diarrhoea & 1 & $25.00 \%$ & 0 & $0.00 \%$ & 3 & $75.00 \%$ & 4 \\
\hline Dysentery & 4 & $66.70 \%$ & 0 & $0.00 \%$ & 2 & $33.30 \%$ & 6 \\
\hline Diarrhoea with malnutrition & 0 & $0.00 \%$ & 0 & $0.00 \%$ & 4 & $100 \%$ & 4 \\
\hline Diarrhoea with other illnesses & 4 & $44.44 \%$ & 0 & $0.00 \%$ & 5 & $55.56 \%$ & 9 \\
\hline Total & 60 & $60.00 \%$ & 2 & $2.00 \%$ & 38 & $38.00 \%$ & 100 \\
\hline
\end{tabular}

Table 4: Average score obtained for 22 criteria of Prescription Quality Index Tool (n=100).

\begin{tabular}{|c|c|c|c|}
\hline Variable & Maximum score & Mean & \pm SD \\
\hline Is there an indication for the drug? & 4 & 0.96 & 1.25 \\
\hline Is the dosage correct? & 4 & 1.53 & 1.90 \\
\hline Is the medication effective for the condition? & 2 & 0.65 & 0.79 \\
\hline Is the usage of drug supported by the evidence? & 2 & 0.56 & 0.72 \\
\hline Are the directions for administration correct? & 2 & 1.97 & 0.171 \\
\hline Are the directions for administration practical? & 2 & 1.96 & 0.24 \\
\hline Are there clinically significant drug-drug interactions? & 2 & 2.00 & 0.00 \\
\hline Are there clinically significant drug-disease/condition interaction? & 2 & 1.98 & 0.20 \\
\hline Does the patient experience any adverse drug reaction(s)? & 2 & 1.94 & 0.31 \\
\hline Is there unnecessary duplication with other drug (s)? & 1 & 0.90 & 0.30 \\
\hline Is the duration of therapy acceptable? & 2 & 0.71 & 0.94 \\
\hline Is this drug the cheapest compared to other alternatives for the same indication? & 1 & 0.28 & 0.45 \\
\hline Is the medication being prescribed by generic name? & 1 & 0.07 & 0.25 \\
\hline Is the medication available in the formulary or essential drug list? & 1 & 0.55 & 0.50 \\
\hline Does the patient comply with the drug treatment? & 2 & 2.0 & 0.0 \\
\hline Is the medication's name on the prescription clearly written? & 2 & 1.89 & 0.34 \\
\hline Is the prescriber's name on the prescription legible? & 2 & 1.88 & 0.35 \\
\hline Is the prescriber's information on the Prescription adequate? & 2 & 2.0 & 0.0 \\
\hline Is the patient's information on the prescription adequate? & 2 & 1.97 & 0.17 \\
\hline Is the diagnosis on the prescription clearly written? & 2 & 1.92 & 0.33 \\
\hline Does the prescription fulfil the patient's Requirement for drug therapy? & 1 & 0.51 & 0.50 \\
\hline Has the patient's condition (s) improved with treatment? & 2 & 2.0 & 0.0 \\
\hline
\end{tabular}


PQI tool assessed whether there was a sufficient reason for the use of drug. Each drug prescribed was evaluated for the indication of the drug and it was observed that $60 \%$ prescriptions had at least one drug which was not indicated as per the recommended WHO guidelines. Only $7 \%$ prescriptions were found to adhere to the WHO criteria in all perspectives.

\section{DISCUSSION}

Children determine an important and a major part of demography in developing countries. They suffer from high morbidity and mortality rate as a result of diarrhoea. Unfortunately, this morbidity and mortality statistics have remained high over a period of time in spite of the medical advancement in availability of medicine. The primary management of diarrhoea aim to focus on restoring the hydration status of the child by paying attention to early rehydration and judicious re-feeding.

In the present study, the male to female ratio was 68:32. A similar pattern was observed in studies conducted in other parts of the country blaming partly for the unfortunate practices for preferring male child over female. ${ }^{8}$ Appropriate management of the child depends on the recognition of the signs of dehydration and managing the patient based on the degree of dehydration. Maximum admissions observed were with no dehydration and with some degree of dehydration $(36 \%)$. This is in contrast to the guidelines that suggest if the child has no signs of dehydration, he/she can be managed at home and parents can be counselled to bring the child to the physician only if signs of dehydration develop. It reflects rather more apprehensive care both from parents as well as from the treating physician. The reason for some admissions could be that out of 36 patients who showed no signs of dehydration, 13 had associated illness in the form of anaemia, febrile seizures, and extra intestinal infection for the remaining $22 \%$ of the admissions with no dehydration no compelling reason was found. Another reason for admission could be that the maximum patients $(81 \%)$ were below the age group of 24 months. Since these are at greater risk of accounting to morbidity and mortality the management was preferred in the hospital setup.

Patients with some dehydration are at relatively greater risk of landing into severe dehydration. In the present study, 36 patients were admitted with some dehydration. Associated illnesses were observed in 16 patients. The magnitude of admission due to severe diarrhoea was only in 5 patients. These findings are contrary to an audit conducted in the year 2016 on prescriptions of 103 patients in the age group of up to 12 years admitted with diarrhoea where maximum admissions were of the patients with some $(50.5 \%)$ dehydration and severe dehydration $(36 \%) .{ }^{9}$ Below $10 \%$ admissions were of the cases of persistent diarrhoea and dysentery in the present study which are similar to a study conducted in Italy in $2014 .{ }^{10}$ Diarrhoea is of great concern when it is associated with severe malnutrition, as the focus is not only at careful management of the nutrition status in addition to the prevention of dehydration, but also at the prevention of systemic infection and fatality associated with it. Underlying malnutrition with diarrhoea was observed in very few cases, as not being a government hospital. This finding was in contrast to a study from other parts of government hospitals in India where the associated malnutrition was observed in relatively more patients. ${ }^{8}$

WHO guidelines recommend the use of ORS and zinc for all children who present with or without some degree of dehydration. Even if the child needs intravenous therapy, he or she should be shifted to oral therapy as soon as the signs of dehydration improve. ORS and zinc were prescribed in $62 \%$ and $81 \%$ of children respectively in the present study. The use of ORS was comparatively more than studies reported from elsewhere in the country but a long journey is yet to be covered in achieving $100 \%$ results. ${ }^{11}$ It has been recorded to be as low as $2 \%$ in the neighbourhood countries, although up to a maximum of $100 \%$ reported earlier in India. ${ }^{9,12}$ In the last national health survey conducted in the state of Uttarakhand, zinc supplementation was observed in $30.4 \%$ of the children. It is quite assuring that zinc prescribing was frequent in this hospital setup.

Clinical guidelines are developed to give recommendations about appropriate health care aim to improve the quality of care. WHO guidelines do not support the use of antiemetic, antacids, anti-diarrhoeal and Pre- and probiotics. Unfortunately, deviation from the protocol was observed in substantial number of patients in this study. Use of probiotics was frequently seen (43\%), although anti-diarrhoeal use was seen in only $6 \%$ patients. Although the values are comparatively less when compared to other study where probiotics was prescribed to $68 \%$ patients and racecadotril to $19 \% .{ }^{13}$ One more study records the use of Pre/probiotics to be $66.6 \% .{ }^{14}$ Deviation was noticed for the use of Multivitamin that were unnecessarily prescribed in 18 patients which is slightly more than earlier data reported in India. ${ }^{8}$

The inpatients in this study were followed up till discharge from the time of admission. As per PQI tool, $10 \%$ of prescription had at least one drug that duplicated in one or other formulation. The duplication was mostly observed for zinc preparations. Similar poor quality of prescription $(36 \%)$ by PQI tool was reported in patients suffering from hypertension and bronchial asthma also in India. ${ }^{15}$ The findings in this study confirms rational approach to the use of ORS and zinc in majority of patients while a huge deviation from the WHO standard guidelines with poor quality of prescription was observed in context of parenteral therapy, antiemetic, probiotics, antidiarrhealland antimicrobials use.

Overall the quality of prescription in the study as observed elsewhere also was poor with utter disregard to adherence to the standard guidelines. A variety of unnecessary drugs were in use that may not have any rationality in treating 
acute and persistent diarrhoea in children up to five years of age. Such studies will definitely expose the pitfalls of present management and it may lay down the path for corrective measures for successful management of such a common disorder of children that is still linked with substantial morbidity and mortality. Still a lot needs to be done to set the standards of treatment in line with WHO standard guidelines for rational prescription in diarrhoeal disorders in children under the age group of five years.

\section{ACKNOWLEDGEMENTS}

The authors acknowledge with gratitude the Himalayan institute of Medical sciences and associated Hospital for providing all support for the conduct of this study.

Funding: No funding sources

Conflict of interest: None declared

Ethical approval: The study was approved by the Institutional Ethics Committee

\section{REFERENCES}

1. Black RE, Cousens S, Johnson HL, Lawn JE, Rudan I, Bassani DG, et al. Global, regional, and national causes of child mortality in 2008: a systematic analysis. The lancet. 2010 Jun 11;375(9730):1969-87.

2. UNICEF. Diarrhoea remains a leading killer of young children, despite the availability of a simple treatment solution. UNICEF data: monitoring the situation of children and women: updated data Mar 2018. Available at: http://data.unicef.org/topic/childhealth/diarrhoeal-disease/\#access_data

3. Sheet IF. NFHS-4 (National Family Health Survey-4). International Institute for Population Studies; 2017.

4. World Health Organization. The treatment of diarrhoea: a manual for physicians and other senior health workers. Geneva. WHO; 2005.

5. Wittenberg, Dankwart F. Management guidelines for acute infective diarrhoea / gastroenteritis in infants. South Afric Med J. 2012;102:104-7.

6. Hassan NB, Ismail HC, Naing L, Conroy RM, Rahman A, Rashid A. Development and validation of a new Prescription Quality Index. Brit J Clin Pharmacol. 2010;70(4):500-13.

7. World health organisation. how to investigate drug use in health facilities; 1993:12-16.
8. Panchal JR, Desai CK, Iyer GS, Patel PP, Dikshit RK. Prescribing pattern and appropriateness of drug treatment of diarrhoea in hospitalized children at a tertiary care hospital in India. Int J Med Public Health. 2013;3(4).

9. De T, Kondekar S, Rathi S. Hospital Based Prospective Observational Study to Audit the Prescription Practices and Outcomes of Paediatric Patients (6 months to 5 years age group) Presenting with Acute Diarrhea. Journal of clinical and diagnostic research. JCDR. 2016 May;10(5):SC01.

10. Vecchio AL, Liguoro I, Bruzzese D, Scotto R, Parola L, Gargantini G, et al. Adherence to guidelines for management of children hospitalized for acute diarrhea. Ped Infect Dise J. 2014 Nov 1;33(11):11038.

11. Lamberti LM, Taneja S, Mazumder S, LeFevre A, Black RE, Walker CL. An external evaluation of the Diarrhea Alleviation through Zinc and ORS Treatment (DAZT) program in Gujarat and Uttar Pradesh, India. J Global Heal. 2015 Dec;5(2).

12. Quadri F, Nasrin D, Khan A, Bokhari T, Tikmani SS, Nisar MI, et al. Health care use patterns for diarrhea in children in low-income periurban communities of Karachi, Pakistan. Ame j Trop Med Hygie. 2013 Jul 10;89(1):49-55.

13. Pathak D, Pathak A, Marrone G, Diwan V, Lundborg CS. Adherence to treatment guidelines for acute diarrhoea in children up to 12 years in Ujjain, India-a cross-sectional prescription analysis. BMC infectious diseases. 2011 Dec;11(1):32.

14. Chakraborti S, Barik KL, Singh AK, Nag SS. Prescribing practices of doctors in management of acute diarrhea. Indian Paediatr. 2011;48(10):811.

15. Suthar JV, Patel VJ, Vaishnav B. Quality of prescribing for hypertension and bronchial asthma at a tertiary health care facility, India using Prescription Quality Index tool. J Basic Clin Pharma. 2014 Dec;6(1):1.

Cite this article as: Sharma N, Dhasmana DC, Sharma T, Gupta A. Quality of prescriptions in hospitalized children suffering from acute and persistent diarrhoea. Int J Basic Clin Pharmacol 2019;8:792-7. 